SUBJECT AREAS:

EVOLUTIONARY THEORY

STATISTICAL PHYSICS

Received

24 September 2014

Accepted

8 December 2014

Published

14 January 2015

Correspondence and requests for materials should be addressed to

R-W.W. (wangrw@ mail.kiz.ac.cn)

* These authors contributed equally to this work. super-rational strategy might resolve the tragedy of the commons without requiring recognition or negotiation

\author{
Jun-Zhou He ${ }^{1,2,3 *}$, Rui-Wu Wang ${ }^{1 *}$, Christopher X. J. Jensen ${ }^{4 *} \&$ Yao-Tang Li ${ }^{5}$
}

\begin{abstract}
'State Key Laboratory of Genetic Resources and Evolution, Kunming Institute of Zoology, Chinese Academy of Science, Kunming, Yunnan, 650223, P.R. China, ${ }^{2}$ Statistics and Mathematics College, Yunnan University of Finance and Economics, Kunming, Yunnan, 650221, P.R. China, ${ }^{3}$ Yunnan Tongchuang Computing \& Data Mining Center, Kunming, Yunnan, 650221, P.R. China, ${ }^{4}$ Department of Mathematics and Science, School of Liberal Arts and Sciences, Pratt Institute, 200 Willoughby Avenue, Brooklyn, NY, USA, ${ }^{5}$ School of Mathematics and Statistics, Yunnan University, Kunming, Yunnan, 650091, P.R. China.
\end{abstract}

Avoiding the tragedy of the commons requires that one or more individuals in a group or partnership "volunteer", benefiting the group at a cost to themselves. Recognition and negotiation with social partners can maintain cooperation, but are often not possible. If recognition and negotiation are not always the mechanism by which cooperative partnerships avoid collective tragedies, what might explain the diverse social cooperation observed in nature? Assuming that individuals interact asymmetrically and that both "weak" and "strong" players employ a super-rational strategy, we find that tragedy of the commons can be avoided without requiring either recognition or negotiation. Whereas in the volunteer's dilemma game a rational "strong" player is less likely to volunteer to provide a common good in larger groups, we show that under a wide range of conditions a super-rational "strong" player is more likely to provide a common good. These results imply that the integration of super-rationality and asymmetric interaction might have the potential to resolve the tragedy of the commons. By illuminating the conditions under which players are likely to volunteer, we shed light on the patterns of volunteerism observed in variety of well-studied cooperative social systems, and explore how societies might avert social tragedies.

C ooperation has been observed in a great variety of human and non-human social systems, attracting the attention of both biologists and social scientists ${ }^{1-4}$. Of particular interest is cooperation that requires voluntary altruistic behavior on the part of one or more members of the group: in order to maintain the benefits of cooperation, individuals may be required to provide a service or resource, refrain from overexploiting a common resource, or to police and punish freeloaders or cheaters ${ }^{1,5-9}$. While such volunteerism contributes to the common good, it comes at a cost to the volunteer, which sets up a dilemma: if not every member of the group volunteers in equal measure, the largest payoffs go to those who volunteer least ${ }^{10,11}$. This inequity discourages volunteerism, leading to the loss of cooperative benefits. This potential for loss has been dubbed the "tragedy of the commons"12, attributed to "diffusion of responsibility"13, and explored as the "freerider problem"14, a "social dilemma"15, and a "multi-person prisoner's dilemma" ${ }^{16,17}$; the diversity of names under which this problem has been investigated underscores the breadth and depth of its importance. Social dilemma research across academic fields is united by the desire to illuminate the conditions under which some or all individuals in a group can be expected to volunteer in support of the common good (i.e. cooperate) rather than refuse to volunteer (i.e. defect).

Two theoretical constructs - the Iterated Prisoner's Dilemma (IPD) and Volunteer's Dilemma game (VoD) have been predominant in research exploring social dilemmas ${ }^{18-24}$. In both the IPD and VoD groups of cooperators outperform groups of defectors, but defectors have the potential to outperform cooperators in groups: this paradox defines the social dilemma. The key difference between these two games is that the probability of cooperation in the IPD is strictly dependent on the cost-to-benefit discount ratio ${ }^{2,25}$ whereas in the VoD the probability of cooperation also depends on the size of the group ${ }^{19,20,26-29}$. In analyzing the outcome of such games, one consideration is whether a "pure" strategy exists which can displace all other strategies. If cooperation is a 
pure strategy, cooperating all of the time should provide the maximum payoff irrespective of what other individuals do. Not surprisingly, pure cooperation is not predicted in social dilemmas such as the IPD ${ }^{30}$ or $\operatorname{VoD}^{19}$. The alternative to a pure strategy is one that is "mixed": individuals employ different strategies with different probabilities. There is strong evidence that in the social systems observed in nature, most animals do not consistently utilize a particular strategy but rather employ different strategies with different probabilities $^{31-33}$. For example, in eusocial colonies (which are defined by a division labor that includes reproductive altruism), ant and bee workers and subordinate mole rats have been shown to exhibit a mixed strategy, altruistically helping most of the time but also infrequently producing their own offspring ${ }^{34,35}$.

The original forms of both the IPD and VoD assume that interactions are symmetrical (i.e. the individual costs and benefits of cooperation are identical for all individuals), which suggests that all individuals should have the same probability of cooperating ${ }^{2,19}$. However, the validity of this assumption has been called into question by a great variety of observational studies that demonstrate that some members of social groups are more likely to volunteer to provide a common good than others ${ }^{5,7,31,34,36,37}$. Because both the IPD and $\mathrm{VoD}$ assume symmetrical interactions, they may not be accurate representations how cooperation occurs in nature. In fact, almost all of the well-studied inter-specific c $^{7,38}$ and intra-specific systems ${ }^{34,35,39}$ have shown that cooperative individuals interact asymmetrically. This asymmetry could be caused by a variety of factors including a difference in resource availability to different individuals, a difference amongst individuals in their probability of winning a fight with others, or other characteristics that differ between dominant and subordinate individuals ${ }^{40}$. Asymmetric interaction alters potential payoffs and therefore may influence cooperative individuals' optimal strategies $^{21,37,40-45}$.

In addition, most analyses of both the VoD and IPD have focused on so-called "rational" solutions to each game, introducing an additional assumption about the drivers of social behavior. A key feature of rational analyses is that they take an individually relativistic approach: an individual will 'rationally' change behavior if that change in behavior affords an advantage over other individuals, even if the result is worse overall for the group. Generally, if freeriding through defection is profitable, it will spread and displace cooperation ${ }^{30,46,47}$. The IPD allows individuals to recognize their social partners and sanction those partners for past defection by withholding future cooperation (a form of costly punishment). While the means of 'communication' between IPD players is restricted to complete information and recognition, these have been shown to be an effective (if not unsophisticated) means of 'negotiating' for future cooperation'.

Frequently, interacting individuals are in an incomplete information state (or have no information, as is sometimes the case in interspecific mutualisms) and may not be able to recognize the identities of other individuals, leaving each individuals to make decisions based solely on their own benefit ${ }^{34,37,48,49}$. An alternative to the rational analysis of evolutionary games is Hofstadter's (1983) "superrational" approach, the theoretical equivalent of Kant's categorical imperative "Act only according to that maxim whereby you can, at the same time, will that it should become a universal law"50,51. Rather than simply seeking the best payoff for themselves, super-rational players pursue the strategy that maximize expected utility when employed by all players.

Do most public goods models predict that a 'tragedy of the commons' will undermine cooperation because their assumptions are at odds with the conditions that actually exist in nature? In this paper we pursue this question by presenting a new model that does not assume symmetrical interaction or rational decision-making and does not rely on individual recognition or negotiation. We build upon previous work that has considered the role of asymmetry in the $\mathrm{VoD}^{26,28}$, the IPD ${ }^{52}$, and other social dilemmas ${ }^{34,36}$ as well as the effect of super-rationality on the outcome of the symmetric $\mathrm{VoD}^{19}$. In presenting the first analysis of the properties of an asymmetric volunteer's dilemma with super-rational players, we suggest a new mechanism by which the 'tragedy of the commons' may be averted.

\section{Model and Results}

The volunteer's dilemma (VoD) is a particular form of public goods game in which at least one member of a social group (henceforth referred to as a "player") must volunteer to pay a cost $(K)$ so that all members of the group (including the volunteer) may enjoy a benefit ( $U$, also referred to as the "common good"). The social dilemma faced by $\mathrm{VoD}$ players is that by volunteering a player will be guaranteed to earn a payoff $(U-K>0)$ but that payoff could be larger ( $U$, so long as at least one other player volunteers) if a player refuses to volunteer. The "tragedy of the commons" occurs when all players refuse to volunteer, hoping that at least one other player will be compelled to provide the common good; if all players refuse to volunteer (adopting the 'freerider' strategy), no one earns a payoff. If players have the option to negotiate, the best outcome for all players in an iterated VoD is to take turns volunteering, but if such negotiation cannot take place (or cannot be enforced) a "tragedy of the commons" outcome is probable ${ }^{19}$.

Because the model presented in this paper is built upon the foundation of previous VoD work, we briefly review the model formulation and results of this previous work below. Those wishing to fully understand these previous analyses should refer to Diekmann (1985, 1993), Archetti (2009) and He et al. (2012) $19,20,26-28$.

Review of relevant previous models of the volunteer's dilemma. An important early analysis of the VoD was conducted by Diekmann (1985) and forms the basis of all models discussed below. In his analysis, Diekmann considered a game composed of $N$ players, all of whom experience the same cost of volunteering $(K)$ and potential for earning the common good $(U)$; because all players experience the same costs and benefits, this version of the VoD is referred to as "symmetric". Using conventional game-theoretic analysis assuming rational players, Diekmann discovered that no dominant strategy exists for the symmetric VoD. And although there is a mixed (i.e. probabilistic) equilibrium strategy, it produces the common good at very low rates. Interestingly, Diekmann considered a different assumption - that of super-rationality - to look for a solution to the symmetric VoD that was more likely to consistently produce the common good; our analysis below will employ an analogous approach on an asymmetric version of the VoD.

Diekmann also produced an analysis of an asymmetric $\mathrm{VoD}$ in a group size $N$ composed of one dominant (hereafter referred to as "strong") and $N-1$ subordinate (hereafter referred to as "weak") players ${ }^{26}$. The asymmetry of the game was manifest in the cost of volunteering to provide a shared common good: the single "strong" player was assumed to pay a lower cost or obtain a greater utility than the "weak" player. Diekmann showed that the expected utility (equivalent to "fitness" in biological terminology) of player $i$ can be expressed in terms of the cost of volunteering $\left(K_{i}\right)$, the benefit earned when at least one player volunteers $\left(U_{i}\right)$, player $i$ 's probability of defection $\left(\beta_{i}\right)$, and the average defection probability of all other players $\left(\beta_{j}\right)$ :

$$
E_{i}=\beta_{i} U_{i}\left(1-\prod_{j \neq i} \beta_{j}\right)+\left(1-\beta_{i}\right)\left(U_{i}-K_{i}\right) .
$$

Diekmann then derived the equilibrium probability of defection for player $i\left(\beta_{i}^{*}\right)$ that maximizes fitness $\left(E_{i}\right)$ :

$$
\beta_{i}^{*}=\frac{U_{i}}{K_{i}}\left(\prod_{j=1}^{N} \frac{K_{j}}{U_{j}}\right)^{\frac{1}{N-1}}
$$

Under the assumptions that the weak players with equal degree of weakness all experience the same cost $\left(K_{W}\right)$ and benefit $\left(U_{W}\right)$, that the common good is the same for all players $\left(U_{S}=U_{W}=1\right)$, and that 
the "strong" player pays a lower cost to volunteer than each of the "weak" players $\left(K_{S}<K_{W}<1\right)$, Diekmann derived from equation (2) the following equilibrium defection of probabilities for "weak" and "strong" players:

$$
\begin{gathered}
\beta_{S}^{*}=\frac{K_{W}}{K_{S}} K_{S^{\frac{1}{N-1}}}, \\
\beta_{W}^{*}=K_{S^{\frac{1}{N-1}}}
\end{gathered}
$$

Where $\beta_{S}^{*}$ and $\beta_{W}^{*}$ are the defection probability of "strong" player and "weak" co-players, and $K_{S}$ and $K_{W}$ are the cooperative cost of "strong" player and "weak" players.

Equations (3-4) make it clear that increasing the group size $(N)$ will lead to increased probabilities of defection for both "strong" and "weak" players. Both of the probabilities also depend on the cost of volunteering to the "strong" player $\left(K_{S}\right)$, but the "strong" player is always predicted to be more likely to defect by a factor of $\frac{K_{W}}{K_{S}}$.

To explore how varying asymmetry affects the defection probabilities of both "strong" and "weak" players, He et al. (2012) extended the analysis of Diekmann (1993) by considering the ratio $\frac{K_{W}}{K_{S}}$ as a new derived parameter, the degree of asymmetry $(h)^{26,28}$. This concept is valuable because it allowed He et al. to more comprehensively explore the role of asymmetry in the $\mathrm{VoD}$, illuminating the conditions under which the "strong" player is predicted to defect with $100 \%$ probability (a contingency of the asymmetric VoD what was not considered by Diekmann (1993)). He et al. demonstrated that there is a critical cost of volunteering $K_{S}^{*}=K_{W}{ }^{(N-1) /(N-2)}$ below which the "strong" player always defects. Accordingly, this also implies that there is a critical degree of asymmetry $h^{*}=K_{W}^{1 /(2-N)}$ above which the "strong" player always defects, a finding consistent with other theoretical analyses ${ }^{44}$. He et al. suggested that because predicted defection probabilities above $100 \%$ have no real meaning (i.e. $\beta_{i}>1$ ), equations (3-4) are more accurately re-written as:

$$
\begin{aligned}
& \beta_{S}^{*}=\left\{\begin{array}{cc}
h\left(\frac{K_{W}}{h}\right)^{\frac{1}{N-1}}, & \left(1<h<h^{*}=K_{W}^{1 /(2-N)}\right) \\
1, & \left(h \geq h^{*}\right)
\end{array}\right. \\
& \beta_{W}^{*}=\left\{\begin{array}{cc}
\left(\frac{K_{W}}{h}\right)^{\frac{1}{N-1}}, & \left(1<h<h^{*}=K_{W}^{1 /(2-N)}\right) \\
\left(K_{W}\right)^{\frac{1}{N-2}}, & \left(h \geq h^{*}\right)
\end{array}\right.
\end{aligned}
$$

Below we will report in more detail what equation (5-6) tell us about the asymmetric $\mathrm{VoD}$, and will compare the equations derived by $\mathrm{He}$ et al. (2012) under the rational assumption with the predictions made when super-rationality is assumed.

Asymmetric volunteer's dilemma game with super-rational strategy. Using Hofstadter's (1983) concept of super-rationality ${ }^{51}$ and building on Diekmann's (1985) application of this concept to the symmetric $\mathrm{VoD}^{19}$, we study the asymmetric VoD with superrational "strong" and N-1 "weak" players. The sole departure of our model from the asymmetric $\mathrm{VoD}$ analyses reviewed above is the assumption of super-rationality. For our version of the asymmetric $\mathrm{VoD}$, equation (1) depicts the fitness of the "strong" $\left(E_{S}\right)$ and $N-1$ "weak" $\left(E_{W}\right)$ players as:

$$
\begin{gathered}
E_{S}=E_{1}=\beta_{S} U_{S}\left(1-\beta_{W}^{N-1}\right)+\left(1-\beta_{S}\right)\left(U_{S}-K_{W}\right) \\
E_{W}=E_{i}=\beta_{W} U_{W}\left(1-\beta_{S} \beta_{W}^{N-2}\right)+\left(1-\beta_{W}\right)\left(U_{W}-K_{W}\right), 2 \leq i \leq N
\end{gathered}
$$

Simultaneous maximization of these expressions yields the superrational strategy ${ }^{53,54}$ :

$$
\begin{gathered}
\beta_{S}^{* *}=\frac{1}{N-1} \frac{K_{W}}{U_{W}} \frac{U_{S}}{K_{S}}\left(\frac{K_{S}}{U_{S}}\right)^{\frac{1}{N-1}} \\
\beta_{W}^{* *}=\left(\frac{K_{S}}{U_{S}}\right)^{\frac{1}{N-1}}
\end{gathered}
$$

where $\beta_{S}^{* *}$ is the expected defection probability of the "strong" player and $\beta_{W}^{* *}$ is the expected defection probability of the $N-1$ "weak" coplayers. Substitution of $\beta_{S}^{* *}$ and $\beta_{W}^{* *}$ in equations (7-8) and some algebraic manipulations yields:

$$
E_{S}^{*}=U_{S}-K_{S}
$$

$$
\begin{aligned}
& E_{W}^{*}=U_{W}-m K_{W}, \\
& \text { where } \frac{1}{N-1}<m<1 \text { and } m=1-\beta_{W}^{* *}\left(1-\frac{1}{N-1}\right)
\end{aligned}
$$

Diekmann (1993) showed that the fitness of rational players is $E_{i}{ }^{*}$ $=U_{i}-K_{i}^{26}$. Comparing this expected fitness to (11-12), it is clear that while the expected fitness of the "strong" player $\left(E_{S}^{*}\right)$ is not changed under the assumption of super-rationality, the "weak" players' fitness is increased (i.e. $E_{W}^{*}=U_{W}-m K_{W}>U_{W}-K_{W}$ ).

To further understand the defection probabilities of "strong" and "weak" players, we let $U_{S}=U_{W}=1$ and maintain the asymmetry between players by positing that $K_{S}<K_{W}<1$. From (9-10) we get:

$$
\begin{gathered}
\beta_{S}^{* *}=\frac{1}{N-1} \frac{K_{W}}{K_{S}} K_{S}^{\frac{1}{N-1}} \\
\beta_{W}^{* *}=K_{S}^{\frac{1}{N-1}}
\end{gathered}
$$

Just as was the case for the asymmetric $\mathrm{VoD}$ with rational players (see equation 3), the result shown in (13) implies that for any cost to the "strong" player in the interval $0<K_{S}<K_{S}^{* *}$ (where $\left.K_{S}^{* *}=\left(K_{W} /(N-1)\right)^{(N-1) / N}\right)$ that player's defection probability will be greater than $100 \%\left(\beta_{S}^{* *}>1\right)$. As discussed in He et al. (2012), we make the realistic assumption that the "strong" player defects with $100 \%$ probability $\left(\beta_{S}^{*}=1\right)$ for all values of $K_{S}<K_{S}^{*}$ once the probability of defection of the "strong" player reaches $100 \%$ (i.e. the "strong" player never volunteers); the probability of the "weak" player is similarly fixed. Under this assumption, the defection probability of the "weak" players is $\left(K_{W} /(N-1)\right)^{\frac{1}{N-2}}$, because the asymmetric game with super-rational strategy becomes a symmetric VoD with $N-1$ "weak" players (as explored by Diekmann 1985). Introducing the degree of asymmetry as $h=\frac{K_{W}}{K_{S}}$, the defection probability of the "strong" player and "weak" players with super-rational strategy becomes:

$$
\begin{gathered}
\beta_{S}^{* *}=\left\{\begin{array}{cc}
\frac{1}{N-1} h\left(\frac{K_{W}}{h}\right)^{\frac{1}{N-1}}, & 1 \leq h \leq h^{* *} \\
1, & h>h^{* *}
\end{array}\right. \\
\beta_{W}^{* *}=\left\{\begin{array}{cc}
\left(\frac{K_{W}}{h}\right)^{\frac{1}{N-1}}, & 1 \leq h \leq h^{* *} \\
\left(\frac{K_{W}}{N-1}\right)^{\frac{1}{N-2}}, & h>h^{* *}
\end{array}\right.
\end{gathered}
$$

where the critical point $h^{* *}=\exp [(N-1) /(N-2)-\ln ((N-1) /$ $\left.\left.K_{W}^{1 /(N-1)}\right)\right]$ (after He et al. 2012).

Having derived the above equations we can now compare the expected outcomes for the asymmetric VoD with rational and super-rational strategies. Figure 1 shows the effects of group size 


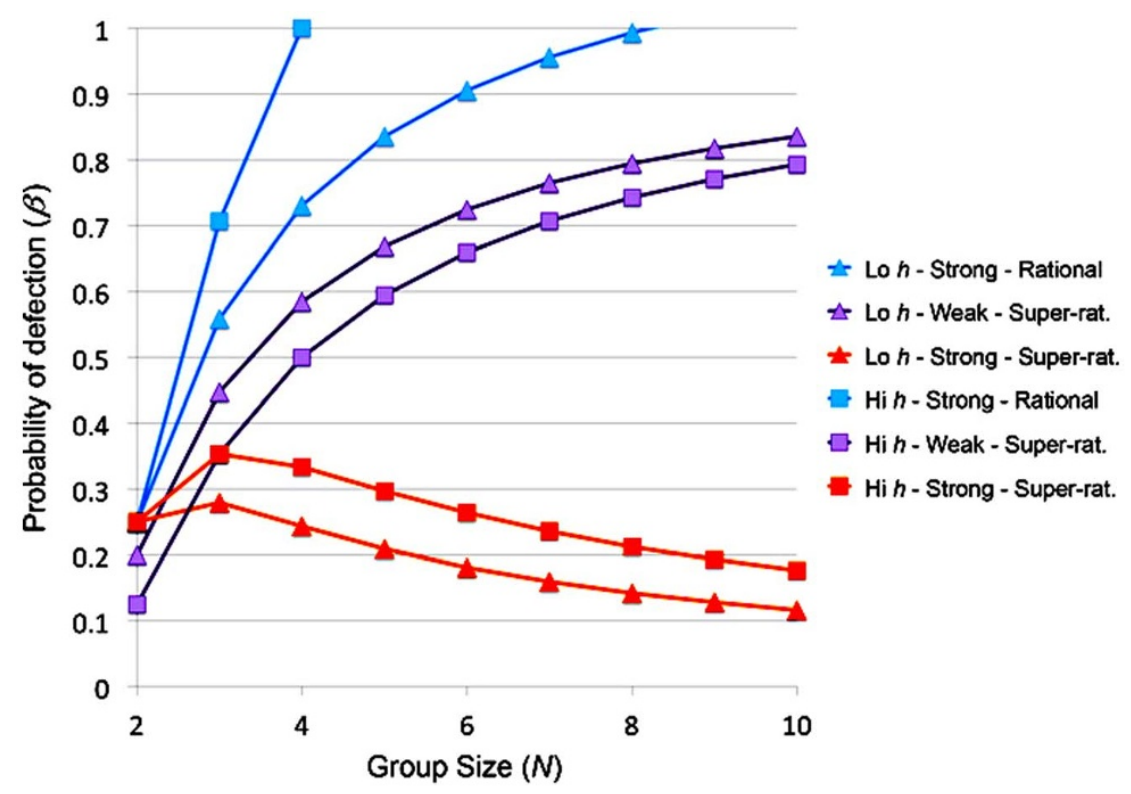

Figure $1 \mid$ Defection probabilities as a function of group size under the assumptions of rationality and super-rationality: Probability of defection ( $\beta$ ) as a function of group size $(N)$ under low (triangles, $h=1.25$ ) and high (squares, $h=2$ ) degrees of asymmetry $(h)$. For the "strong" player, defection probabilities vary greatly depending on whether the strategy is assumed to be rational (blue shapes based eq. 5) or super-rational (red shapes based on eq. 15). For the "weak" player, defection probabilities (purple shapes) are comparable regardless of whether or not the strategy assumed is rational or super-rational. In this figure, we show only the super-rational "weak" players" defection probabilities (purple shapes based on eq. 16); the rational defection probabilities are identical until the "strong" player's defection probability reaches $100 \%\left(\beta_{s}=1\right)$, at which point the "weak" player's defection probability is fixed (see eq. 6). For all plots the cooperative cost to "weak" players is fixed at $K_{w}=0.25$.

$(N)$ and degree of asymmetry $(h)$ on the defection probability $(\beta)$ of "strong" and "weak" players. As shown previously by Diekmann (1993), the probability of defection $(\beta)$ of all rational players increases as group size $(N)$ increases, with $\beta_{S}{ }^{*}>\beta_{W}{ }^{*}$ for all values of $N^{26}$. As shown previously by He et al. (2012), higher degrees of asymmetry $(h)$ lead to larger differences between the defection probabilities of "strong" and "weak" players ${ }^{28}$. What is new in Figure 1 is the result for super-rational players: while at low group sizes the "strong" player is more likely to defect, at a critical group size $N^{*}$ the "strong" player's defection probability begins to decrease, which at larger group sizes means that $\beta_{W}{ }^{*}>\beta_{S}{ }^{* *}$ (see Supplementary Information (SI) for a formal derivation of $\left.N^{*}\right)$. As implied by the equivalence of (6) and (16), the defection probabilities of superrational and rational "weak" players are the same at lower degrees of asymmetry $\left(h<h^{*}\right.$ and $\left.h<h^{* *}\right)$. While higher degrees of asymmetry $(h)$ lead to larger differences in the defection probabilities of rational "strong" and "weak" players, the introduction of superrationality produces the opposite trend: higher degrees of asymmetry (h) lead to smaller differences in defection probabilities.

Figure 2 further explores the effect increasing degrees of asymmetry $(h)$ on the defection probabilities $(\beta)$ of "strong" and "weak" players. As previously shown by $\mathrm{He}$ et al. (2012) and displayed in Figure $2 \mathrm{~A}$, increasing asymmetry in the rational $\mathrm{VoD}$ leads to more dramatic differences in the defection probabilities $(\beta)$ of "strong" and "weak" players, and above the critical degree of asymmetry $\left(h^{*}\right)$ the "strong" player always defects (which reduces the game to a symmetric VoD between "weak" players, see above) ${ }^{28}$. In contrast, the current analysis of the super-rational asymmetric VoD makes more complex predictions about the defection probabilities $(\beta)$ of "strong" and "weak" players (Figure 2B). While there still exists a critical degree of asymmetry above which the "strong" player always defects $\left(h^{* *}\right)$, the asymmetry must be much larger in order to remove the "strong" player from the pool of potentially-volunteering players (i.e. $h^{* *}>h^{*}$ ). In addition, as Figure 2B demonstrates, the defection probability of the "strong" player is much lower than that of the "weak" player for low degrees of asymmetry $(h<h$ ').
Figure 3 provides a more comprehensive representation of superrational findings already provided in Figures 1 and 2 by plotting the probability of cooperation for the "strong" player $\left(\beta_{S}{ }^{* *}\right)$ and "weak" players $\left(\beta_{W^{* *}}\right)$ across a wide range of group sizes $(N)$ and degrees of asymmetry $(h)$. Figure $3 \mathrm{~A} / \mathrm{B}$ demonstrates that the super-rational findings shown in Figures 1 and 2 are robust over a large range of model parameters.

Production of the common good in variations of the volunteer's dilemma. The tragedy of the commons is averted when a common good is produced. In this section we examine how different VoD assumptions affect the probability $(P)$ that this common good is produced. In our simple version of the $\mathrm{VoD}$ only one player is required to volunteer in order to produce the collective good, which means that $P=1-\prod_{i=1}^{N} \beta_{i}^{* 26}$.

As shown by Diekmann $(1993)^{26}$, the probability that the common good will be produced in the asymmetric version of the VoD that assumes that players are rational can be expressed as:

$$
\begin{aligned}
& P_{A R}=1-\beta_{S}^{*}\left(\beta_{W}^{*}\right)^{N-1} \\
& =\left\{\begin{array}{cc}
1-\frac{K_{W}}{U_{W}}\left(\frac{K_{S}}{U_{S}}\right)^{\frac{1}{N-1}}, & \left(K_{S} \geq K_{S}^{*}=K_{W}^{(N-1) /(N-2)}\right) \\
1-K_{W}^{\frac{N-1}{N-2}}, & \left(K_{S}<K_{S}^{*}\right)
\end{array}\right.
\end{aligned}
$$

Under the assumptions of asymmetry and super-rationality employed in our VoD model, the probability of producing the common good can be expressed as:

$$
\begin{aligned}
& P_{A S}=1-\beta_{S}^{* *}\left(\beta_{W}^{* *}\right)^{N-1} \\
& =\left\{\begin{array}{cc}
1-\frac{1}{N-1} \frac{K_{W}}{U_{W}}\left(\frac{K_{S}}{U_{S}}\right)^{\frac{1}{N-1}}, & \left(K_{S} \geq K_{S}^{* *}=\left(K_{W} /(N-1)\right)^{(N-1) / N}\right)_{(18)} \\
1-\left(\frac{K_{W}}{N-1}\right)^{\frac{N-1}{N-2}}, & \left(K_{S}<K_{S}^{* *}\right)
\end{array}\right.
\end{aligned}
$$




\section{A Rational strategy B Super-rational strategy}

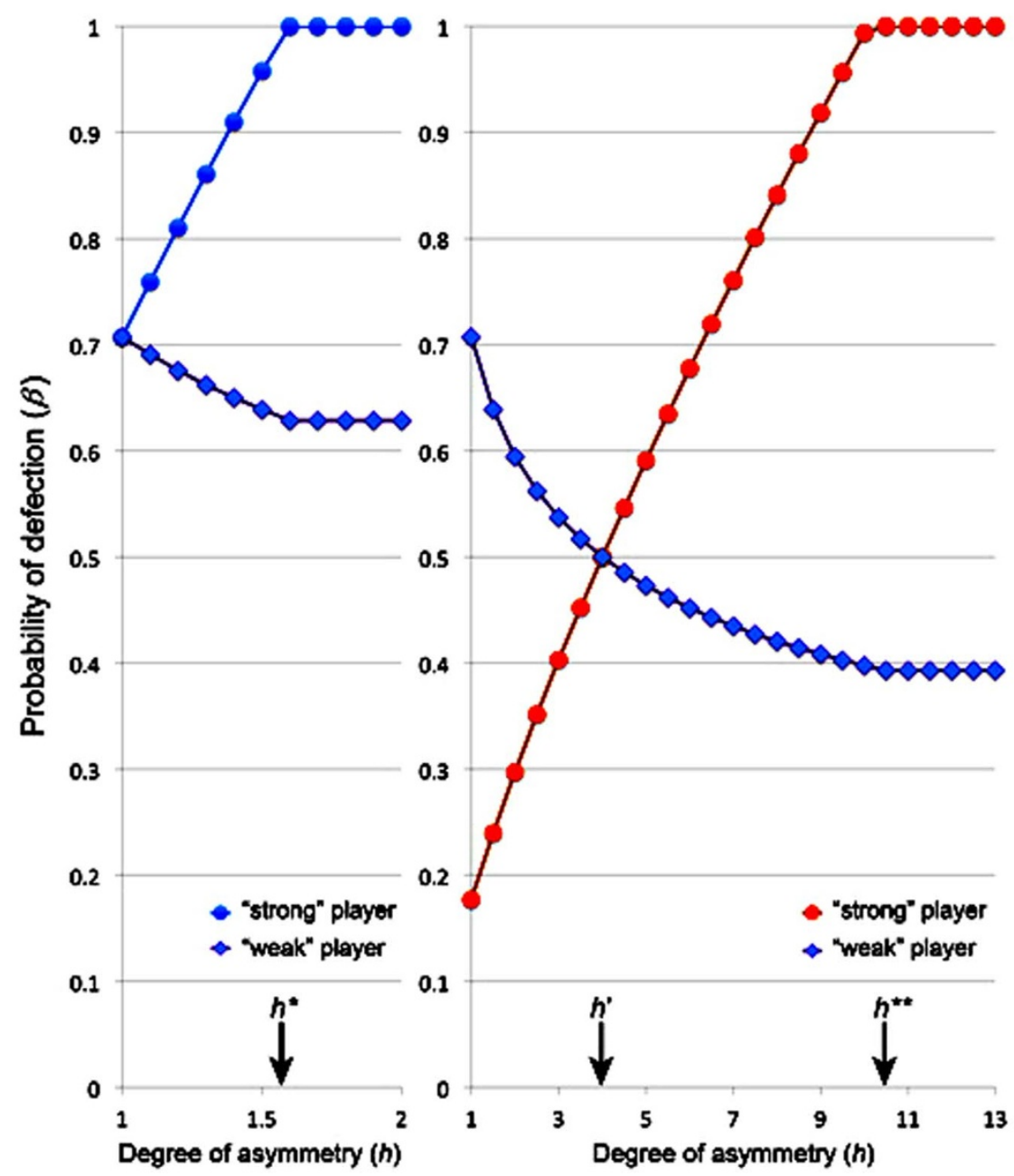

Figure $2 \mid$ Defection probabilities as a function of asymmetry under the assumptions of rationality and super-rationality: Probability of defection $(\boldsymbol{\beta})$ as function of degree of asymmetry $(h)$ for strong (circles) and weak (diamonds) players. For "strong" players, the probability of defection is strongly affected by whether strong players are assumed to adopt the rational (A, blue circles based on eq. 5) or the super-rational (B, red circles based on eq. 15) strategy. For "weak" players the probability of defection under the rational assumption (A, purple triangles based on eq. 6) only differs from the super-rational assumption (B, purple triangles based on eq. 16) based on when the "strong" player is predicted to defect with $100 \%$ probability. Three critical values of the degree of asymmetry $(h)$ are shown with arrows: when $h \geq h^{*}$ the rational "strong" player always defects, when $h=h$ ' the super-rational players have equal defection probabilities, and when $h \geq h^{* *}$ the super-rational "strong" player always defects. For all plots the cooperative cost to "weak" players is fixed at $K_{w}=0.25$ and group size is fixed at $N=5$.

For convenience, we let $U_{S}=U_{W}=1, K_{S}<K_{W}<1$ ( $K_{W}$ be constant) and $K_{W} / K_{S}=h$ (the degree of asymmetry), which produces the following:

$$
P_{A R}=\left\{\begin{array}{cc}
1-K_{W}\left(\frac{K_{W}}{h}\right)^{\frac{1}{N-1}}, & \left(h \leq h^{*}=K_{W}{ }^{1 /(2-N)}\right) \\
1-K_{W^{\frac{N-1}{N-2}}} & \left(h>h^{*}\right)
\end{array}\right.
$$

$P_{A S}=$

$\left\{\begin{array}{cc}1-\frac{K_{W}}{N-1}\left(\frac{K_{W}}{h}\right)^{\frac{1}{N-1}}, & \left(h \leq h^{* *}=\exp \left[\frac{N-1}{N-2}-\ln \frac{N-1}{K_{W}{ }^{1 /(N-1)}}\right]\right)_{(20)} \\ 1-\left(\frac{K_{W}}{N-1}\right)^{\frac{N-1}{N-2}}, & \left(h>h^{* *}\right)\end{array}\right.$

Figure 4 demonstrates how the probability of producing the common good $(P)$ is affected by variations in the degree of asymmetry $(h)$ and group size $(N)$ for asymmetric versions of the VoD considered above. Under the rational assumption the probability of producing the common good $(P)$ decreases with increasing group size $(N)$ when interactions are asymmetrical (red squares representing $P_{A R}$, whose limit value is $1-K_{W}$ ); that is, for larger groups with a high cost of volunteering the tragedy of the commons is more likely to occur in the VoD when rational strategies are employed. A comparison of Figure 4A $(h=1.25)$ with Figure $4 \mathrm{~B}(h=2)$ demonstrates that greater degrees of asymmetry increase the likelihood of producing the common good (red squares representing $P_{A R}$ and blue diamonds representing $\left.P_{A S}\right)$ in both the rational and super-rational scenarios. In contrast, introduction of the super-rational strategy (Figure 4, blue diamonds representing $P_{A S}$ ) can - above a critical group size $N^{*}$ (see Supplementary Information SI) - leads to increased common good production with increased group size. The limit value of $P_{A S}$ is 1 , which implies that for larger groups the tragedy of the commons can be averted so long as the cost of volunteering is lower than the common benefit. As with the rational version of the asymmetric VoD, a comparison of Figure 4A $(h=1.25)$ with Figure 4B $(h=2)$ 
A Probability of cooperation for the "strong" player

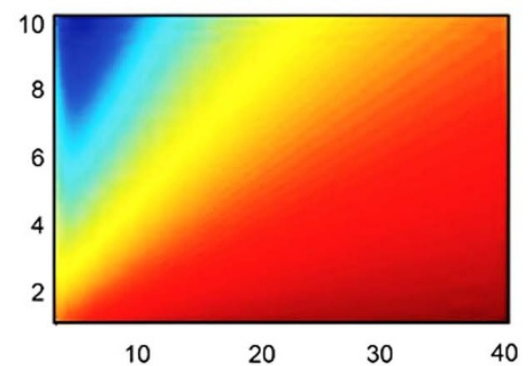

C Probability of producing the collective good

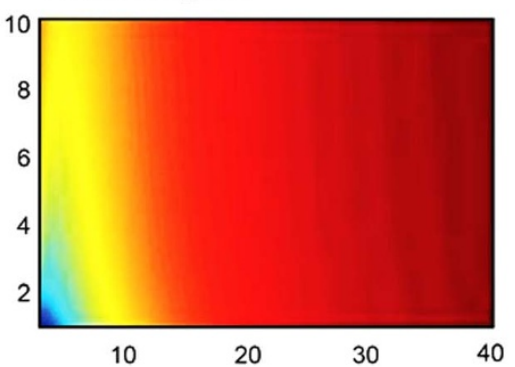

B Probability of cooperation for the "weak" players

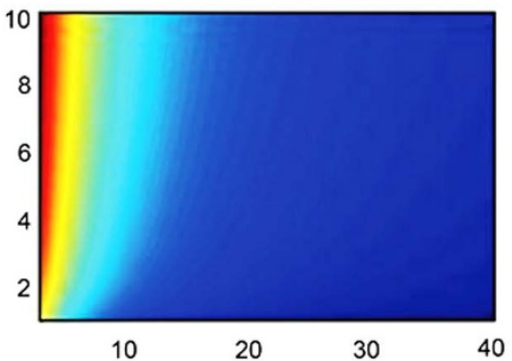

Key:

(for all plots)

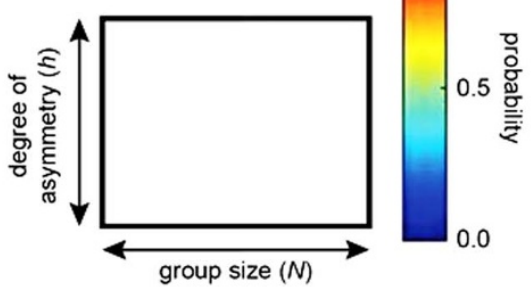

Figure 3 Collective good production in the asymmetric volunteer's dilemma game (super-rational solution): The probability of cooperation for the "strong" (A) and "weak" players (B) and the probability of producing the collective good (C) are shown as functions of the degree of asymmetry $(h)$ and the group-size $(N)$. Plots are based on equations $15(\mathrm{~A}), 16(\mathrm{~B})$, and 20 (C) with the cooperative cost to weak players fixed at $K_{w}=0.2$.

demonstrates that greater degrees of asymmetry increase the likelihood of producing the common good when super-rationality is assumed $\left(P_{A S}\right)$. Figure $3 \mathrm{C}$ provides a more comprehensive repres- entation of super-rational findings already provided in Figure 4 by plotting the probability of producing the common good $\left(P_{A S}\right)$ across a wide range of group sizes $(N)$ and degrees of asymmetry $(h)$.
A Lower degree of asymmetry $(h=1.25)$

\section{B Higher degree of asymmetry $(h=2)$}
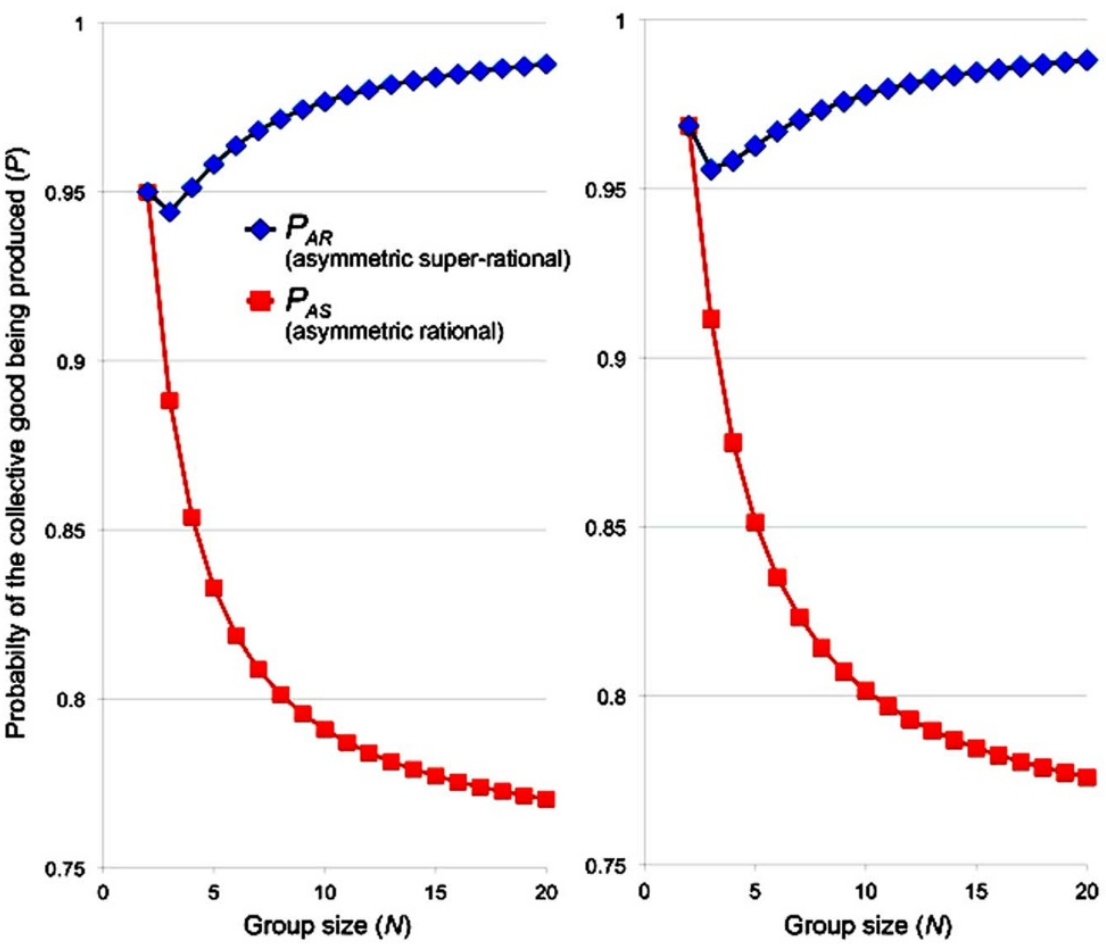

Figure 4 Collective good production in various forms of the volunteer's dilemma (VoD) game: The probability of producing the collective good $(P)$ as a function of the group size $(N)$ in the, asymmetric VoD assuming a rational strategy (red squares based on eq. 19) and the asymmetric VoD assuming a super-rational strategy (blue diamonds based on eq. 20). Results are shown for low $(\mathrm{A}, h=1.25)$ and high $(\mathrm{B}, h=2)$ levels of asymmetry. For all plots the cooperative cost to "weak" players is fixed at $K_{w}=0.25$. 
Figure $3 \mathrm{C}$ demonstrates that the super-rational findings shown in Figure 4 are robust over a large range of model parameters.

\section{Discussion}

Diekmann (1993) has pointed out that the predicted behavior of "strong" players in the asymmetric VoD depends on the method of game analysis employed ${ }^{26}$. The mixed Nash-equilibrium, which represents a "rational" analysis, predicts that the "strong" player will be more likely to defect than the $N-1$ "weak" players (as in our Figure 1 and Figure 2A, blue lines compared to purple lines). Other theories for finding stable strategies in games, including the Harsanyi-Selten theory ${ }^{53}$ and Schelling's "prominent" solution ${ }^{55}$, make the opposite prediction ${ }^{26}$. We add to Diekmann's analysis by showing that when the "strong" and "weak" players both employ a super-rational strategy, the probability of defection of the "strong" player $\left(\beta_{S}\right)$ is predicted to be lower than the "weak" players within a particular range of asymmetry (Figure $2 \mathrm{~B}$, when $h<h^{\prime}$ ), and decreases as group size $(N)$ increases (as in Figure 1, red lines compared to purple lines, Figure 4A/B, and Figure 3A). This shift in the behavior of the "strong" player leads to increased likelihood of producing the common good (Figure 3C), making our model the first version of the $\mathrm{VoD}$ that demonstrates a means by which the tragedy of the commons can be consistently averted.

The model developed here demonstrates that, when interactions are asymmetric, the tragedy of the commons can be avoided even when interacting individuals are simply maximizing their own fitness or income gain; complicated maintenance mechanisms such as recognition or negotiation are not necessarily required. In systems with weak or non-existent recognition (such as in microbial systems or in interspecific mutualisms), individuals are more likely to adopt a strategy that depends only on an assessment of what produces their best absolute outcome (i.e. the super-rational strategy). For example, leguminous host plants reduce or increase oxygen supplies (which in turn decreases or increases the growth rate of symbiotic bacteria) in response to the amount of nitrogen provided by rhizobia in the root nodule ${ }^{5}$. Yucca plants also abort the fruits oviposited by moths based on how many seeds they produced ${ }^{48}$. These systems stand in contrast to human cooperative systems in which individuals are often able to effectively communicate with each other (i.e. are in a "complete information state") and public goods can be produced by rational individuals through partner recognition and/or negotiation mechanisms ${ }^{56-60}$.

Assuming that recognition and negotiation are not possible, and considering asymmetric contributions to the common good which have been observed in humans ${ }^{2}$, bees ${ }^{35}$, mole-rats ${ }^{34}$, and a number of inter-specific mutualisms $s^{32,37,47,61}$, the model developed here demonstrates that the 'strong' player is more likely to produce a common good in increasingly large groups if the degree of asymmetry is lower than the critical degree of asymmetry $\left(h^{* *}\right)$ (Figure 3A, red region). In a cooperative system composed of many individuals, free-riding 'weak' individuals are less likely to encounter 'strong' players and therefore less likely to be punished by these 'strong' players ${ }^{1}$. Absent the ability to effectively utilize punishment as an enforcement strategy $^{62}$, the 'strong' players are more likely prevent the loss of cooperative benefits -- as observed in the model developed here -- because in maintaining cooperation 'strong' players stand to gain much greater benefits than 'weak' players ${ }^{63}$. However, in cooperative systems composed of only a few individuals, the freeriding 'weak' individuals will be more likely to encounter the 'strong' players and be punished. The weaker individuals therefore should be more likely to pay the cost to provide the common good; this explains the observed results of our model that when the group size is small and the degree of asymmetry is higher than the critical degree of asymmetry $\left(h^{* *}\right)$, the 'strong' player tends to defect but the common good is likely to be produced by 'weak' players (Figure 3B; Figure 4B, red region).

Asymmetry distinguishes our model from most of the theoretical work that has been presented to resolve the tragedy of the commons ${ }^{64,65}$.
Many animal societies have been shown to be composed of dominant and subordinate individuals ${ }^{66-68}$, whose behavioral options may be well-represented by our "strong" and "weak" players. The degree of asymmetry between dominant and subordinate individuals varies, and in our model there exists a critical degree of asymmetry $(h ')$ below which the dominant individual is more likely to volunteer. Lower degrees of asymmetry may explain why adult meerkats (Suricata surucatta), are more likely to produce alarm calls than juveniles $^{69}$. Perhaps the most fitting comparison of our model is to eusocial insect colonies in which eggs produced by 'cheating' workers are in some species removed by the queen ${ }^{39,70}$ and in other species removed by the workers ${ }^{71}$. Our model suggests that removal by the queen should occur in species where social asymmetry is mild, whereas removal by workers should occur when the social asymmetry is much greater; this explanation has the potential to be investigated empirically.

Most theoretical explorations of the tragedy of the commons are designed to explain intra-specific cooperation. While our model might explain why members of the same species with asymmetric social interactions cooperate, it also can be applied to inter-specific mutualisms, which are inherently asymmetrical. Our assumption of super-rationality applies particularly well to inter-specific mutualisms because interacting members of different species are usually not in competition with each other and therefore gain nothing from the relativistic logic of rational behavior. Super-rationality also applies well to mutualist species because they depend on the success of their partner species in order to survive and reproduce. Nonetheless, cheating can occur in mutualisms ${ }^{72}$, and our model sheds light on which species should volunteer to prevent cheating. Plants often pay costs in mutualistic interactions (suggesting that they are the "strong" player), and have been shown to volunteer to prevent cheating in leguminous plants that host nitrogen-fixing bacteria ${ }^{5}$ and yucca ${ }^{48,61}$ and fig ${ }^{47}$ species that abort fruits in which insect pollinators have 'cheated' by over-exploitation and/or under-pollination.

Can our model be applied to humans? Humans do seem to consistently depart from a "rational" approach. Many human social dilemmas - such as fisheries overexploitation or anthropogenic climate change - emerge in situations where negotiation between large groups has proved unfeasible. In addition, social asymmetry is a common element within most human societies as well as being a global inter-societal phenomenon. But despite these parallels with the model presented here, human societies cannot be fully represented by such a simple model because they are composed of a variety of social groups operating at a diversity of scales and organizational structures $^{73}$. Where would our model apply? Experimental studies of the $\mathrm{VoD}$ suggest that increasing the cost of volunteering leads to less rather than more volunteerism ${ }^{74}$, a result that is inconsistent with previous VoD analyses ${ }^{26}$ but is well-explained by our super-rational asymmetric VoD. Such experimental studies suggest that in smallscale social groups humans may apply a super-rational approach.

Perhaps the most meaningful comparison of our model to actual human societies would be at the largest scale of social organization, that of nation-states. Nation-states aggregate the interests of incredibly large numbers of individuals, simultaneously representing and influencing their populations through national policies. As such, nation-states may act as 'individuals' interacting in a 'community of nations'. Clearly these interactions are not symmetrical: some nation-states have a greater potential to provide for the common good than others. Interactions between nation-states can sometimes involve negotiation, but when it comes to the most problematic ecological tragedies of the commons (e.g. fisheries collapse and destructive climate change), negotiation has often proved ineffective $e^{75,76}$. Perhaps, if negotiations continue to fail, nations with the greater ability to make sacrifices (i.e. "strong" player nations) will decide to pay the cost of preventing fisheries collapses or mitigating climate change. To more realistically represent the interactions of 
nation-states, future modeling efforts must consider the impact of more than one "strong" player or varying degrees of asymmetry between players on overall volunteerism in the social group. To better apply to human social dilemmas in general, future modeling should also consider the role that asymmetry and super-rationality might play in the multiple levels of human social organization.

In conclusion, introducing a super-rational strategy radically changes the predicted outcome of the asymmetric volunteer's dilemma (VoD). Whereas VoD models assuming that players employ rational strategies predict that the individual probability of volunteerism will decrease in larger social groups, we show that as long as the asymmetry between the single "strong" and multiple "weak" players is not too extreme and the group is sufficiently large, the super-rational strategy compels "strong" players to volunteer more frequently in larger groups. This increased volunteerism from "strong" players translates to greater overall cooperation (as measured by the probability of producing the common good) in larger groups experiencing intermediate degrees of asymmetry, a result that is consistent with observational studies performed on both human and non-human animals. Future theoretical and empirical work should focus on the evolutionary mechanisms that might allow a super-rational strategy to persist in the face of cheating, free-riding, and defection.

1. Frank, S. A. Foundations of social evolution. (Princeton University Press, 1998).

2. Axelrod, R. The Evolution of Cooperation. (Basic Books, 1984).

3. Perc, M. \& Szolnoki, A. Coevolutionary games-a mini review. BioSystems 99, 109-125 (2010).

4. Perc, M., Gómez-Gardeñes, J., Szolnoki, A., Floría, L. M. \& Moreno, Y. Evolutionary dynamics of group interactions on structured populations: a review. J R Soc Interface 10, 20120997 (2013).

5. Kiers, E. T., Rousseau, R. A., West, S. A. \& Denison, R. F. Host sanctions and the legume-rhizobium mutualism. Nature 425, 78-81 (2003).

6. Wang, R. W., Shi, L., Ai, S. M. \& Zheng, Q. Trade-off between reciprocal mutualists: local resource availability-oriented interaction in fig/fig wasp mutualism. J. Anim. Ecol. 77, 616-623 (2008).

7. Wang, R. W. et al. Interference competition and high temperatures reduce the virulence of fig wasps and stabilize a fig-wasp mutualism. PLoS One 4, e7802 (2009).

8. Perc, M. Success-driven distribution of public goods promotes cooperation but preserves defection. Phys Rev E 84, 037102 (2011).

9. Wang, Z., Szolnoki, A. \& Perc, M. Evolution of public cooperation on interdependent networks: The impact of biased utility functions. EPL (Europhysics Letters) 97, 48001 (2012).

10. Zhang, H.-F., Liu, R.-R., Wang, Z., Yang, H.-X. \& Wang, B.-H. Aspirationinduced reconnection in spatial public-goods game. EPL (Europhysics Letters) 94, 18006 (2011).

11. Wang, Z., Szolnoki, A. \& Perc, M. Percolation threshold determines the optimal population density for public cooperation. Phys Rev E 85, 037101 (2012).

12. Hardin, G. The tragedy of the commons. Science 162, 1243-1248 (1968).

13. Darley, J. M. \& Latane, B. Bystander intervention in emergencies: diffusion of responsibility. J J. Pers. Soc. Psychol. 8, 377-383 (1968).

14. Dunbar, R. I. M. Culture, honesty and the freerider problem. (Edinburgh University Press, 1999).

15. Dawes, R. M. Social Dilemmas. Annu. Rev. Psychol. 31, 169-193 (1980).

16. Wang, Z., Szolnoki, A. \& Perc, M. Self-organization towards optimally interdependent networks by means of coevolution. New Journal of Physics 16, 033041 (2014)

17. Wang, Z., Szolnoki, A. \& Perc, M. Rewarding evolutionary fitness with links between populations promotes cooperation. J Theor Biol 349, 50-56 (2014).

18. Axelrod, R. \& Hamilton, W. D. The evolution of cooperation. Science 211, 1390-1396 (1981)

19. Diekmann, A. Volunteer's dilemma. J. Conflict. Resol. 29, 605-610 (1985).

20. Archetti, M. The volunteer's dilemma and the optimal size of a social group. J. Theor. Biol. 261, 475-480 (2009).

21. Nikiforakis, N., Normann, H. T. \& Wallace, B. Asymmetric enforcement of cooperation in a social dilemma. Southern Economic Journal 76, 638-659 (2010).

22. Wang, Z., Szolnoki, A. \& Perc, M. If players are sparse social dilemmas are too: Importance of percolation for evolution of cooperation. Scientific reports 2, 369 (2012)

23. Wang, Z., Szolnoki, A. \& Perc, M. Optimal interdependence between networks for the evolution of cooperation. Scientific reports 3, 2470 (2013).

24. Wang, Z., Szolnoki, A. \& Perc, M. Interdependent network reciprocity in evolutionary games. Scientific reports 3, 1183 (2013).
25. Nowak, M. A. \& May, R. M. Evolutionary games and spatial chaos. Nature 359, 826-829 (1992).

26. Diekmann, A. Cooperation in an asymmetric volunteer's dilemma game theory and experimental evidence. Int. J. Game Theory 22, 75-85 (1993).

27. Archetti, M. Cooperation as a volunteer's dilemma and the strategy of conflict in public goods games. J. Evol. Biol. 22, 2192-2200 (2009).

28. He, J. Z., Wang, R. W., Christopher, X. J. J., Li, Y. T. \& Li, C. Q. Cooperation in an asymmetric volunteer's dilemma game with relatedness. Chin. Sci. Bull. 57, 1972-1981 (2012).

29. Szolnoki, A. \& Perc, M. Group-size effects on the evolution of cooperation in the spatial public goods game. Phys Rev E 84, 047102 (2011).

30. Boyd, R. \& Lorberbaum, J. P. No Pure Strategy Is Evolutionarily Stable in the Repeated Prisoners-Dilemma Game. Nature 327, 58-59 (1987).

31. Heinsohn, R. \& Packer, C. Complex cooperative strategies in group-territorial African lions. Science 269, 1260-1262 (1995).

32. Wang, R. W. \& Shi, L. The evolution of cooperation in asymmetric systems. Sci. China Life Sci. 53, 139-149 (2010).

33. Szolnoki, A. \& Perc, M. Conditional strategies and the evolution of cooperation in spatial public goods games. Phys Rev E 85, 026104 (2012).

34. Reeve, H. Queen activation of lazy workers in colonies of the eusocial naked molerat. Nature 358, 147-149 (1992).

35. Rankin, D. J., Bargum, K. \& Kokko, H. The tragedy of the commons in evolutionary biology. Trends Ecol. Evol. 22, 643-651 (2007).

36. Ratnieks, F. L. W. \& Wenseleers, T. Altruism in insect societies and beyond: voluntary or enforced? Trends Ecol. Evol. 23, 45-52 (2007).

37. Wang, R. W., Sun, B. F., Zheng, Q., Shi, L. \& Zhu, L. Asymmetric interaction and indeterminate fitness correlation between cooperative partners in the fig-fig wasp mutualism. J. R. Soc. Interface 8, 1487-1496 (2011).

38. Pellmyr, O. \& Leebens-Mack, J. Reversal of mutualism as a mechanism for adaptive radiation in yucca moths. Am. Nat. 156, S62-S76 (2000).

39. Wenseleers, T. \& Ratnieks, F. L. W. Comparative analysis of worker reproduction and policing in eusocial hymenoptera supports relatedness theory. Am. Nat. 168, E163-E179 (2006).

40. He, J.-Z., Wang, R.-W. \& Li, Y.-T. Evolutionary Stability in the Asymmetric Volunteer's Dilemma. Plos One 9, e103931 (2014).

41. Maynard Smith, J. Evolution and the theory of games. (Cambridge University Press, 1982).

42. Grafen, A. The logic of divisively asymmetric contests: respect for ownership and the desperado effect. Anim. Behav. 35, 462-467 (1987).

43. Abramson, G., Soto, C. A. T. \& Oña, L. The role of asymmetric interactions on the effect of habitat destruction in mutualistic networks. Plos One 6, e21028 (2011).

44. Szolnoki, A. \& Perc, M. Impact of critical mass on the evolution of cooperation in spatial public goods games. Phys Rev E 81, 057101 (2010).

45. Helbing, D. \& Johansson, A. Evolutionary dynamics of populations with conflicting interactions: Classification and analytical treatment considering asymmetry and power. Phys Rev E 81, 016112 (2010).

46. Hauert, C. \& Doebeli, M. Spatial structure often inhibits the evolution of cooperation in the snowdrift game. Nature 428, 643-646 (2004).

47. Wang, R. W., Sun, B. F. \& Zheng, Q. Diffusive coevolution and mutualism maintenance mechanisms in a fig-fig wasp system. Ecology 91, 1308-1316 (2010).

48. Huth, C. \& Pellmyr, O. Pollen-mediated selective abortion promotes evolutionary stability of mutualism between yuccas and yucca moths. Ecology 81, 1100-1107 (2000).

49. Wang, R. W., He, J. Z., Wang, Y. Q., Shi, L. \& Li, Y. T. Asymmetric interaction will facilitate the evolution of cooperation. Sci. China Life Sci. 53, 1041-1046 (2010).

50. Kant, I. Grounding for the Metaphysics of Morals. (Hackett Publishing Company, Inc, 1785).

51. Hofstadter, D. The calculus of cooperation is tested through a lottery. Sci. Am. 6, 14-18 (1983)

52. Du, W. B., Cao, X. B. \& Hu, M. B. The effect of asymmetric payoff mechanism on evolutionary networked prisoner's dilemma game. Phys. A Stat. Mech. Appl. 388, 5005-5012 (2009).

53. Harsanyi, J. C. \& Selten, R. A general theory of equilibrium selection in games (M.I.T. Press, 1988)

54. Binmore, K. G. Playing fair: Game theory and the social contract. (MIT Press, 1994).

55. Schelling, T. C. The strategy of conflict. (Harvard University Press, 1960).

56. Beecher, M. D., Beecher, I. M. \& Hahn, S. Parent-offspring recognition in bank swallows: II. Development and acoustic basis. Anim. Behav. 29, 95-101 (1981).

57. Davis, L. S. \& McCaffrey, F. T. Recognition and parental investment in adelie penguins. Emu 89, 155-158 (1989).

58. Hepper, P. G. Kin recognition. (Cambridge University Press, 2005).

59. Komdeur, J. \& Hatchwell, B. Kin recognition: function and mechanism in avian societies. Trends Ecol. Evol. 14, 237-241 (1999).

60. Taylor, P. D. \& Day, T. Stability in negotiation games and the emergence of cooperation. Proc. R. Soc. B-Biol. Sci. 271, 669-674 (2004).

61. Pellmyr, O. \& Huth, C. J. Evolutionary stability of mutualism between yuccas and yucca moths. Nature 372, 257-260 (1994)

62. Szolnoki, A. \& Perc, M. Correlation of positive and negative reciprocity fails to confer an evolutionary advantage: Phase transitions to elementary strategies. Phys.l Rev. X 3, 041021 (2013). 
63. Frank, S. A. Policing and group cohesion when resources vary. Anim. Behav. 52, 1163-1169 (1996).

64. Ng, C., Wang, Y. \& Zhao, T. Joint Effects of Asymmetric Payoff and Reciprocity Mechanisms on Collective Cooperation in Water Sharing Interactions: A Game Theoretic Perspective. Plos One 8, e73793 (2013).

65. Wang, Y.-L. Asymmetric evaluation of fitness enhances spatial reciprocity in social dilemmas. Chaos, Sol. Frac. 54, 76-81 (2013).

66. Rubenstein, D. R. \& Shen, S. F. Reproductive conflict and the costs of social status in cooperatively breeding vertebrates. Am. Nat. 173, 650-662 (2009).

67. Ang, T. Z. \& Manica, A. Aggression, segregation and stability in a dominance hierarchy. Proc. R. Soc. B-Biol. Sci. 277, 1337-1343 (2010).

68. Clutton-Brock, T. H., Hodge, S. J., Flower, T. P., Spong, G. F. \& Young, A. J. Adaptive Suppression of Subordinate Reproduction in Cooperative Mammals. Am. Nat. 176, 664-673 (2010).

69. Hollén, L. I., Clutton-Brock, T. \& Manser, M. B. Ontogenetic changes in alarm-call production and usage in meerkats (Suricata suricatta): adaptations or constraints? Behav. Ecol. Sociobiol. 62, 821-829 (2008).

70. Liebig, J., Monnin, T. \& Turillazzi, S. Direct assessment of queen quality and lack of worker suppression in a paper wasp. Proc. R. Soc. B-Biol. Sci. 272, 1339-1344 (2005).

71. Ratnieks, F. L. W. \& Visscher, P. K. Worker policing in the honeybee. Nature 342 , 796-797 (1989).

72. Gersani, M., Brown, J. S., O’Brien, E. E., Maina, G. M. \& Abramsky, Z. Tragedy of the commons as a result of root competition. J. Ecol. 89, 660-669 (2001).

73. Folke, C., Hahn, T., Olsson, P. \& Norberg, J. Adaptive governance of socialecological systems. Annu. Rev. Environ. Resour 30, 441-473 (2005).

74. Healy, A. \& Pate, J. in 18th World IMACS/MODSIM Congress 1457-1462 (Cairns, Australia 2009)

75. Webster, D. The irony and the exclusivity of Atlantic bluefin tuna management. Marine Policy 35, 249-251 (2011).

76. Roberts, J. T. Multipolarity and the new world (dis) order: US hegemonic decline and the fragmentation of the global climate regime. Glob. Environ. Change 21, 776-784

\section{Acknowledgments}

We thank Ya-Qiang Wang and Lei Gao for their discussion and comments. This research was supported by the National Natural Science Foundation of China (31370408,31270433, $31170408,71161020,10961027)$, the National Science Fund for Distinguished Young Scholars (31325005), NSFC-Yunnan United fund (U1302267), the West Light Foundation of the Chinese Academy of Sciences, Yunnan Natural Science Foundation (2013FD030), Special Fund for Chair Professor Lei Shi, Special Fund for Provincial Key DisciplinesStatistics, the Program for Innovative Research Team (in Science and Technology) in University of Yunnan Province.

\section{Author contributions}

Conceived and designed the model: R.W.W., Y.T.L. and J.Z.H. Performed the research: J.Z.H. and Y.T.L. Analyzed the model: J.Z.H. and C.X.J.J. Wrote the paper: J.Z.H., R.W.W. and C.X.J.J.

\section{Additional information}

Supplementary information accompanies this paper at http://www.nature.com/ scientificreports

Competing financial interests: The authors declare no competing financial interests.

How to cite this article: He, J.-Z., Wang, R.-W., Jensen, C.X.J. \& Li, Y.-T. Asymmetric interaction paired with a super-rational strategy might resolve the tragedy of the commons without requiring recognition or negotiation. Sci. Rep. 5, 7715; DOI:10.1038/srep07715 (2015)

This work is licensed under a Creative Commons Attribution-NonCommercialNoDerivs 4.0 International License. The images or other third party material in this article are included in the article's Creative Commons license, unless indicated otherwise in the credit line; if the material is not included under the Creative Commons license, users will need to obtain permission from the license holder in order to reproduce the material. To view a copy of this license, visit http:// creativecommons.org/licenses/by-nc-nd/4.0/ 